\title{
THROUGHPUT ESTIMATION WITH REGARD TO AIRTIME CONSUMPTION UNFAIRNESS IN MIXED DATA RATE WI-FI NETWORKS
}

The paper discusses throughput unfairness inherent in the very nature of mixed data rate Wi-Fi networks employing random media access control technique CSMA/CA. This unfairness exhibits itself through the fact that slow clients consume more airtime to transfer a given amount of data, leaving less airtime for other clients. This decreases the overall network throughput and significantly degrades performance of high data rate clients. In the paper we propose mathematical models considering airtime unfairness and estimating wireless networks throughput depending on number of network connections and their data rates. These models show that all wireless clients have an equal throughput independently of data rates used by them. We verify our theoretical findings by running natural experiment and show that client's throughput approximates to the data rate of the slowest client.

Keywords: Wireless networks, Wi-Fi, throughput, airtime consumption, unfairness.

\section{Introduction}

Ethernet family of networks uses the CSMA/CD (Carrier Sense Multiple Access with Collision Detection) media access control technique, which provides random pseudo-fair access to the media for multiple users. This technique has been enhanced toward decreasing the probability of collision in wireless communications. This technique which is a core of IEEE $802.11 \mathrm{a} / \mathrm{b} / \mathrm{g} / \mathrm{n}$ Wi-Fi networks is called Carrier Sense Multiple Access with Collision Avoidance (CSMA/CA) [1].

In general case CSMA provides a random pseudo-fair multiply access to a wireless media that means a statistically equal number of chances that each computer gets to transfer its data frames over a shared media. For instance, if there are five computers connected to the same Fast Ethernet hub, the throughput available to the each computer can be roughly estimated as (Throughput_MAX)/(the number of devices), i.e. $100 / 5=20$ Mbps.

In contrast with the majority of network technologies worked over cables, the data rate used by Wi-Fi devices is not a constant value. Depending on signal strength (signal to noise ratio) a wireless access point (AP) and its clients, i.e. associated stations (STAs), are handshaking about the maximal possible transmitting/receiving data rate. The farther away a client is from the access point or from another client in case of Ad-hoc networks, the weaker a signal or the stronger a noise, the lower is the data rate.

802.11 devices, depending on the number of spatial streams and on the width of the wireless channel and guard interval, support rates from 6.5 Mbps up to $600 \mathrm{Mbps}$. Various data rates are represented by a Modulation and Coding Scheme (MCS) index value (1..31) which also defines modulation schemes and coding rates used.

The situation when different clients connected to the same AP (or sharing the same wireless channel in case of Ad-hoc communications) use different data rates is widespread. Slow clients occupy more airtime to transfer a frame of the same size, significantly degrading the performance of high-speed clients in the network. These facts significantly complicate estimation of the throughput available for a particular wireless client especially in case when all clients are busy transmitting and receiving data.

In this paper we focus on estimating throughput unfairness existing in wireless networks incorporating both low and high data rate clients. This is one of the most topical and yet not effectively resolved performance issues in mixed Wi-Fi networks occurred when high-speed 802.11 clients have to share $802.11 \mathrm{n}$ access points together with the legacy $802.11 \mathrm{a} / \mathrm{b} / \mathrm{g}$ clients [2]. Besides, this problem arises when one of the wireless clients is forced to reduce its data rate because of the weak strength of

\footnotetext{
* ${ }^{1}$ Alaa Mohammed Abdul-Hadi, ${ }^{1}$ Olga Tarasyuk, ${ }^{1}$ Anatoliy Gorbenko, ${ }^{1}$ Vyacheslav Kharchenko, ${ }^{2}$ Thomas Hollstein

${ }^{1}$ Department of Computer Systems and Networks, National Aerospace University “KhAI”, Kharkiv, Ukraine, E-mail: kharchenko@khai.edu

${ }^{2}$ Department of Computer Engineering, Tallinn University of Technology, Tallinn, Estonia
} 
a signal (caused by the significant remoteness from the AP) or considerable noise level.

The paper introduces analytical models assessing existing unfairness towards high speed clients and overall throughput degradation as functions of a given divergence between clients' data rates in a mixed wireless network. Our theoretical findings have been verified by the practical throughput measurement.

There have been several studies of experimental measurement [3 and 4] and analytical estimation [5 and 6] of wireless networks throughput. These studies aim at analyzing Wi-Fi performance and understanding why the real throughput is significantly less than the advertised speed of wireless technologies (up to $54 \mathrm{Mbps}$ for $802.11 \mathrm{a} / \mathrm{g}$ and up to $600 \mathrm{Mbps}$ for $802.11 \mathrm{n}$ ).

Even though these works are important for understanding CSMA/CA performance overheads and uncovering the nature of the performance unfairness in mixed Wi-Fi networks, they do not provide a mathematical apparatus for its estimation.

The rest of the paper is organized as follows. In the next section we investigate a phenomenon of the airtime consumption unfairness arisen in mixed data rate wireless networks. Section 3 introduces analytical models quantifying existed unfairness and estimating wireless network throughput depending on the number of wireless clients and data rates they use. Finally, we verify our theoretical findings by running natural experiment and performing practical measurement, whose results are reported in Section 4, and conclude our work in section 5.

\section{Airtime Consumption Unfairness}

Slow clients consume more airtime to transfer a given amount of data, leaving less airtime for other clients. This decreases the overall network throughput and significantly degrades performance of high data rate clients.

Figure 1 helps in understanding the nature of this problem. It shows the simplified airtime distribution for the following scenarios:

1) there is only one high data rate client $C_{1}$ occupying the whole airtime;

2) there are two high data rate clients $C_{1}$ and $C_{2}$ sharing the wireless media and transferring frames of the same size with the same data rates $\left(\mathrm{V}_{1}=\mathrm{V}_{2}\right)$;

3) there are two clients $C_{1}$ and $C_{2}$ sharing a wireless media and transferring frames of the same size. The data rate $\mathrm{V}_{2}$ of the second client is twice as less as the data rate $\mathrm{V}_{1}$ of the first client $\left(\mathrm{V}_{2}=\mathrm{V}_{1} / 2\right)$;

4) the data rate $V_{2}$ of the second client $C_{2}$ is three times less than the data rate $V_{1}$ of the first client $C_{1}\left(V_{2}=V_{1} / 3\right)$;

5) the data rate $V_{2}$ of the second client $C_{2}$ is five times less than the data rate $\mathrm{V}_{1}$ of the first client $\mathrm{C}_{1}\left(\mathrm{~V}_{2}=\mathrm{V}_{1} / 5\right)$;

6) the data rate $V_{2}$ of the second client $C_{2}$ is eleven times less than the data rate $\mathrm{V}_{1}$ of the first client $\mathrm{C}_{1}\left(\mathrm{~V}_{2}=\mathrm{V}_{1} / 11\right)$.
The inter-frame gap and the contention window can be neglected as their durations are much smaller than the frame transmission time. Besides, Fig. 1 assumes an alternate access to the media by different clients that is possible in theory but cannot be guaranteed in practice if CSMA/CA is used.

As it can be seen from Fig. 1, though the fast client could have sent more frames during the time the slow client spent sending only one, it still has to compete fairly for the wireless media on a frame-by-frame basis. Thus, the high data rate client spends more time waiting for the slow client to release the media than transmitting its own frames. This means that even a single client connected to the wireless network at a low data rate can dramatically slow down all of the high-speed clients.

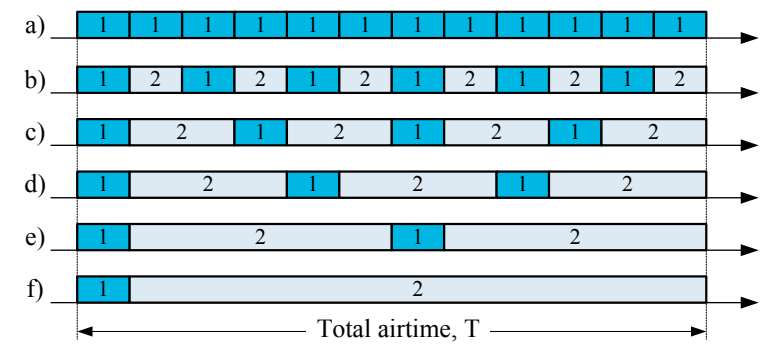

Fig. 1 CSMA/CA airtime distribution between two clients depending on their data rates

\section{Throughput Estimation with Regard to Airtime Consumption Unfairness}

If there is only one client connected to the wireless network at the data rate $\mathrm{V}_{1}$ (see Fig. 1a) the volume of information that can be sent by this client during the given time $\mathrm{T}$ is equal to

$$
N=V_{1} \cdot T
$$

In case of two clients a portion of airtime $T_{1}$ utilized by a client connected at the data rate $\mathrm{V}_{1}$ decreases inversely as the ratio between data rates (we assume that both clients transmit data using frames of the same MTU which is equal to $2.3 \mathrm{Kbytes}$ in case of 802.11 wireless networks):

$$
T_{1}=T \cdot V_{2} /\left(V_{1}+V_{2}\right)
$$

Thus, the volume of information that can be sent by the first client with the data rate $V_{1}$ during the time $T_{1}$ is equal to

$$
N_{1}=V_{1} \cdot T_{1}=T \cdot V_{1} \cdot V_{2} /\left(V_{1}+V_{2}\right),
$$

which actually has the same value for the second client too. This means that the effective throughput $U_{1}$ of the first client is $\mathrm{K}_{1}$ times less compared to its data rate $\mathrm{V}_{1}$ : 


$$
U_{1}=V_{1} / K_{1}
$$

where $K_{1}$ (a degradation coefficient) is equal to a ratio between $\mathrm{N}_{1}$ and $\mathrm{N}_{1}$ ':

$$
K_{1}=N / N_{1}=\left(V_{1}+V_{2}\right) / V_{2} .
$$

Hence,

$$
U_{1}=V_{1} \cdot V_{2} /\left(V_{1}+V_{2}\right)
$$

Using the same reasoning, the throughput $\mathrm{U}_{2}$ of the second client is decreased by $\mathrm{K}_{2}$ times as compared to its data rate $\mathrm{V}_{2}$ :

$$
\begin{aligned}
& K_{2}=\left(V_{1}+V_{2}\right) / V_{1}, \\
& U_{2}=V_{2} / K_{2}=V_{2} \cdot V_{1} /\left(V_{1}+V_{2}\right) .
\end{aligned}
$$

As it can be seen from (6) and (8) both clients get the same throughput though their data rates are different. We can also conclude that a faster client experiences much more significant throughput degradation while the throughput of the slow client is almost equal to its data rate.

Table 1 shows the differences between the degradation coefficients $\left(\mathrm{K}_{1}\right.$ and $\left.\mathrm{K}_{2}\right)$ and the throughput depending on the data rates $\left(V_{1}\right.$ and $\left.V_{2}\right)$ of two clients sharing the same wireless media. It is clear, that the random CSMA/CA access control technique significantly decreases effectiveness of the mixed Wi-Fi networks and is unfair towards clients with the higher data rate.
When two clients use the same data rates they share out network throughput equally as a half of their data rate (see the last row of Table 1). The bigger the difference between data rates used by clients, the lower the throughput they have and the closer it approximates to the data rate of the slowest client. For instance (see the first row of Table 1), when the first client is connected to a wireless network at $300 \mathrm{Mbps}$ whereas the second one uses 15 Mbps connection a throughput available to both clients is equal to $14.3 \mathrm{Mbps}$.

By analogy with (1) - (6) a degradation coefficient $\mathrm{K}_{1}$ in case of the three clients connected to the wireless network at data rates $\mathrm{V}_{1}, \mathrm{~V}_{2}$ and $\mathrm{V}_{3}$ can be estimated as

$$
K_{1}=\frac{V_{2} \cdot V_{3}+V_{1} \cdot V_{3}+V_{1} \cdot V_{2}}{V_{2} \cdot V_{3}} .
$$

Hence, client throughput is equal to

$$
U_{1}=U_{2}=U_{3}=\frac{V_{1} \cdot V_{2} \cdot V_{3}}{V_{2} \cdot V_{3}+V_{1} \cdot V_{3}+V_{1} \cdot V_{2}} .
$$

Generalizing described particular cases we can get universal analytical equations which estimate maximal theoretical clients throughput and degradation factor in case of $n$ clients sharing a wireless media at different data rates:

$$
\begin{aligned}
& K_{i}=V_{i} \cdot \sum_{j=1}^{n} \frac{1}{V_{j}}, \\
& U=U_{i}=\frac{\alpha}{\sum_{j=1}^{n} \frac{1}{V_{j}}},
\end{aligned}
$$

Table 1

Throughput unfairness in mixed Wi-Fi networks

\begin{tabular}{|c|c|c|c|c|c|c|}
\hline \multicolumn{2}{|c|}{ MSC index } & \multicolumn{2}{c|}{ Data rate, Mbps } & \multicolumn{2}{c|}{ Degradation coefficient } & Throughput, Mbps \\
\hline MSC $_{1}$ & $\mathrm{MSC}_{2}$ & $\mathrm{~V}_{1}$ & $\mathrm{~V}_{2}$ & $\mathrm{~K}_{1}$ & $\mathrm{~K}_{2}$ & $\mathrm{U}_{1}=\mathrm{U}_{2}$ \\
\hline 15 & 0 & 300 & 15 & 21.00 & 1.05 & 14.29 \\
\hline 15 & 1 & 300 & 30 & 11.00 & 1.10 & 27.27 \\
\hline 15 & 2 & 300 & 45 & 7.67 & 1.15 & 39.13 \\
\hline 15 & 3 & 300 & 60 & 6.00 & 1.20 & 50.00 \\
\hline 15 & 4 & 300 & 90 & 4.33 & 1.30 & 69.23 \\
\hline 15 & 5 & 300 & 120 & 3.50 & 1.40 & 85.71 \\
\hline 15 & 6 & 300 & 135 & 3.22 & 1.45 & 93.10 \\
\hline 15 & 7 & 300 & 150 & 3.00 & 1.50 & 100.00 \\
\hline 15 & 8 & 300 & 30 & 11.00 & 1.10 & 27.27 \\
\hline 15 & 9 & 300 & 60 & 6.00 & 1.20 & 50.00 \\
\hline 15 & 10 & 300 & 90 & 4.33 & 1.30 & 69.23 \\
\hline 15 & 11 & 300 & 120 & 3.50 & 1.40 & 85.71 \\
\hline 15 & 12 & 300 & 180 & 2.67 & 1.60 & 112.50 \\
\hline 15 & 13 & 300 & 240 & 2.25 & 1.80 & 133.33 \\
\hline 15 & 14 & 300 & 270 & 2.11 & 1.90 & 142.11 \\
\hline 15 & 15 & 300 & 300 & 2.00 & 2.00 & 150.00 \\
\hline
\end{tabular}


where $a$ is OSI Level 7 throughput efficiency coefficient.

Many practical studies, e.g. [3 and 4], showed that a throughput practically achieved at the OSI Level-7 (or even Level-4) is substantially lower than the data rate at which a client is connected to the wireless network at level 1. It takes about 50\% at best even in case of a single client connected to an access point and it gets worse when there are multiple clients sharing the same wireless network.

In [6] an efficiency coefficient $\alpha(0<a \leq 1)$ has been introduced to take into account such a decrease caused by many reasons including inter-frame gaps and CSMA/CA contention windows, numerous $\mathrm{Wi}-\mathrm{Fi}$ control frames, collisions and retransmissions of corrupted frames. More detailed explanations of this phenomenon are given in [7], but they are out of the scope of this paper. Moreover, if a wireless network works in an Infrastructure mode every communication between wireless clients goes through the access point. This means that the same message goes over the air twice (from the source STA to the $\mathrm{AP}$ and then from the AP to the destination STA) and leaving only half of the available throughput. Thus, a value of the Level 7 throughput efficiency coefficient $\alpha$ can be roughly estimated using Table 2 taking into account different types of WLAN and communication.

Finally, the overall bandwidth of a wireless network incorporating $n$ associated stations connected at different data rates can be estimated as:

$$
U_{\Sigma}=\alpha \cdot n \cdot U_{i}=\frac{\alpha \cdot n}{\sum_{j=1}^{n} \frac{1}{V_{j}}} .
$$

WLAN throughput degradation coefficient

Table 2

\begin{tabular}{|l|l|l|}
\hline $\begin{array}{l}\text { Service Set/Type } \\
\text { of WLAN }\end{array}$ & $\begin{array}{l}\text { Type of } \\
\text { Communication }\end{array}$ & $\begin{array}{l}\text { Throughput degradation } \\
\text { coefficient } a\end{array}$ \\
\hline IBSS/Ad-hoc & WLAN $\longleftrightarrow$ WLAN & $0.3-0.5$ \\
\hline \multirow{2}{*}{ BSS/Infrastructure } & WLAN $\longleftrightarrow$ WLAN & $0.15-0.25$ \\
\cline { 2 - 3 } & WLAN $\longleftrightarrow$ LAN & $0.3-0.5$ \\
\hline
\end{tabular}

\section{Experimental Throughput Measurement}

\subsection{Method and Experimental Settings}

The experiments that we report in this section use the configuration depicted in Fig. 2. It includes one desktop computer C connected directly to the 802.11 n MIMO UniFi AP access point via $100 \mathrm{Mbps}$ Fast Ethernet wired connection. Besides, we used two laptops (A and B) equipped with the Intel WiFi Link 6250 and Atheros AR9285 802.11n network adapters respectively. These laptops established network connections with the desktop computer C via the DAP-1360/E 802.11n access point. Each laptop supported one spatial downstream ( 1 x $2: 1)$ using one 20-MHz-wide channel in the $2.4 \mathrm{GHz}$ range. Both laptops were configured to simultaneously establish TCP connections with the desktop computer and to send 100 files of $1 \mathrm{MB}$ size each in series.

At the beginning both laptops were placed close to AP so that were able to establish wireless connections at the maximal available data rates: 75.2 Mbps for A and $65 \mathrm{Mbps}$ for B. This initial difference of the data rates is caused by the different guard intervals supported by Intel and Atheros adapters (400 ns and $800 \mathrm{~ns}$ ). While a position of the laptop B was fixed during the whole experiment, the laptop A had been moved off the access point until its data rate decreased down to the minimal value ( 6.5 Mbps). Then it was moved back toward the access point (see Fig. 2).

To run this experiment and to measure network throughput and signal strength (RSSI) we used IxChariot tool which is the industry's leading test tool for simulating real-world applications and measuring system performance under realistic load conditions ${ }^{1}$.

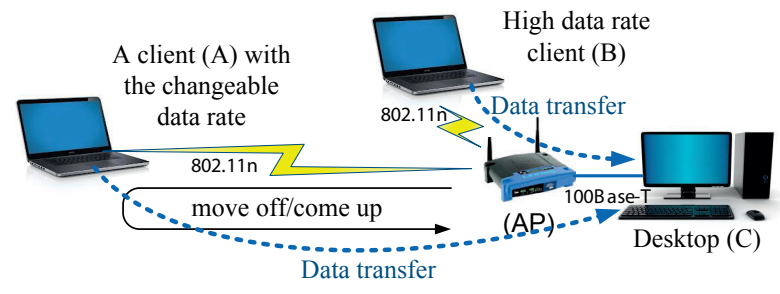

Fig. 2 Experimental configuration

\subsection{Signal Strength and Throughput Measurement Results}

As we can see in Fig. 3a the RSSI (Received Signal Strength Indication) measured by the laptop B was varying insignificantly during the whole observation time whereas the RSSI measured by the laptop A was decreasing when it was moving away from the access point and increasing back when it was coming up. As it also follows from Fig. 3a the receiver sensitivity of the laptop A was a bit better than one of the laptop B.

RSSI decrease caused reduction of the transmission data rate at the laptop A. As a result, its throughput went down as it is shown in Fig. 3b. At the same time we can see that the throughput of the laptop B went down too, though its data transfer rate remained constant during the whole experiment. This testifies to our theoretical findings about the throughput unfairness existing in mixed data rate Wi-Fi networks which is caused by the fact that slow clients consume more airtime to transfer a given amount of data leaving less for others.

However, we can see that throughput curves of the two clients are not exactly the same. This can be explained by the random nature of the CSMA/CA media access control technique and

1 http://www.ixchariot.com/products/datasheets/ixchariot.html 
differences in hardware/software implementation of Intel and Atheros Wi-Fi adapters used by different clients.

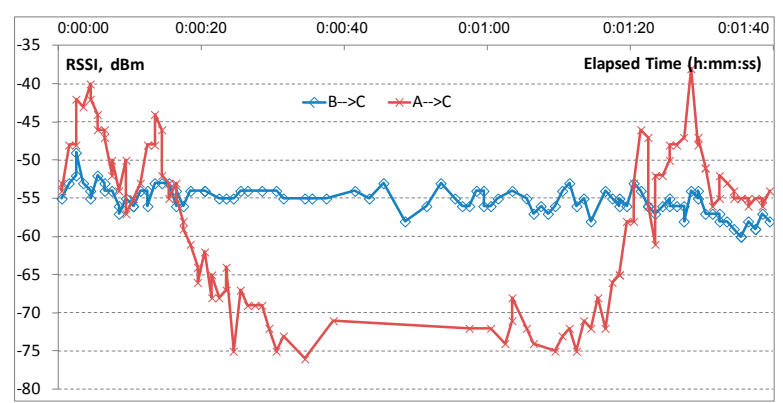

(a)

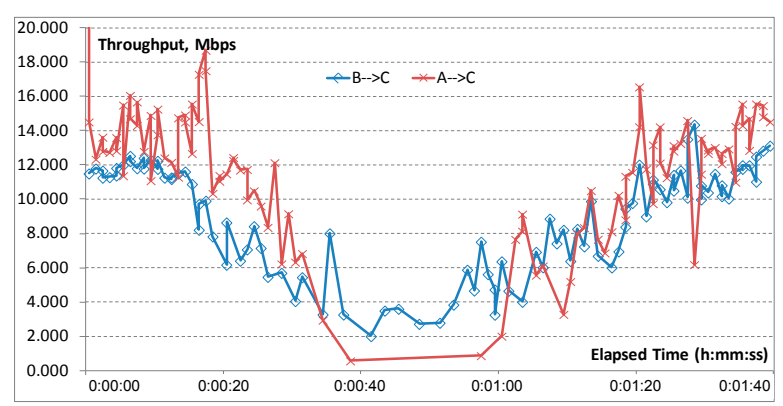

(b)

Fig. 3 Measurement results: received signal strength indication (a) and throughput (b)

\subsection{Deviation Between the Theoretical and Experimental Results}

With the purpose of estimating accuracy of the throughput analytical model (12) let us consider three time intervals observed during the experiment (see Fig. 3): [0:00:00 ... 0:00:10], [0:00:40 ... 0:00:50], and [0:01:30 ... 0:01:40].
During the first and the third time intervals clients A and B transferred data at their maximal data rates (75.2 Mbps for $\mathrm{A}$ and $65 \mathrm{Mbps}$ for B). Thus, we can join these two intervals together (see Table 3). The average Client A's throughput measured during these time intervals was equal to $13.93 \mathrm{Mbps}$. The average Client B's throughput was a bit lower (11.7 Mbps). Using these data we were able to estimate the Level 7 efficiency coefficient $\alpha$ which in our experiments approximated to 0.373 , which is in line with other studies [3 and 4].

During the second time interval we registered the maximal difference between data rates used by clients $\mathrm{A}$ and $\mathrm{B}$ (6.5 Mbps for A and 65 Mbps for B). At the same time their average throughput was equal to $1.62 \mathrm{Mbps}$ (A) and $2.94 \mathrm{Mbps}$ (B) respectively.

Client's maximal theoretical throughput estimated using (12) and assuming $\alpha=0.373$ is equal to $12.66 \mathrm{Mbps}$ for the first and the third time intervals. For the second time intervals it is equal to $2.19 \mathrm{Mbps}$. As it can be seen, the deviation between results obtained theoretically and those average values measured practically is less than $4 \%$ for the second time interval and $0.5 \%$ for the first and third intervals. Such a convergence between theoretical and experimental results approves accuracy of our analytical models proposed in section 3 .

\section{Conclusions}

In general case CSMA/CA provides a random multiply access to a wireless media that means a statistically equal number of chances that each computer gets to transfer its data frames over a shared media. However, different wireless computers can use different data rates depending on the signal-to-noise ratio. This fact causes an unfairness as slow clients consume more airtime to transfer a given amount of data, leaving less airtime for other clients.

\begin{tabular}{|c|c|c|}
\hline \multirow[b]{2}{*}{ Parameter } & \multicolumn{2}{|c|}{ Time interval, h:mm:ss } \\
\hline & $\begin{array}{l}{[0: 00: 00 \ldots 0: 00: 10]} \\
{[0: 01: 30 \ldots 0: 01: 40]}\end{array}$ & {$[0: 00: 40 \ldots 0: 00: 50]$} \\
\hline Client A Data Rate & 72.20 & 6.50 \\
\hline Client B Data Rate & 65.00 & 65.00 \\
\hline Client A experimental throughput (average) & 13.93 & 1.62 \\
\hline Client B experimental throughput (average) & 11.70 & 2.94 \\
\hline Level 7 throughput degradation coefficient $\alpha$ & \multicolumn{2}{|c|}{0.373} \\
\hline $\begin{array}{l}\text { Theoretical clients throughput estimated using (12) and } \\
\text { assuming efficiency coefficient } \alpha=0.373\end{array}$ & 12.76 & 2.20 \\
\hline Average clients experimental throughput & 12.82 & 2.28 \\
\hline Deviation & $0.44 \%$ & $3.44 \%$ \\
\hline
\end{tabular}


At the same time, high data rate clients spent much more of air time just waiting for an access to the wireless media than transferring data. This decreases the overall network throughput and significantly degrades performance of high data rate clients. In the paper we investigate this phenomenon and propose mathematical models which estimate wireless connection throughput taking into account such unfairness occurred in mixed data rate Wi-Fi networks. We have shown both theoretically and experimentally that high data rate clients suffer much more from performance degradation than those connected at low data rate. Finally, all wireless clients have roughly the same throughput independently of data rates used by them. In the case when slow and high-speed clients are simultaneously sharing a wireless media their throughputs approximate to the data rate of the slowest client. Thus, CSMA/CA media access control technique needs to be improved in order to prevent wireless networks from performance degradation.

We have foreseen several approaches which can be used to deal with the throughput degradation caused by the airtime consumption unfairness. The first one is based on the frame aggregation that is an intrinsic part of the $802.11 \mathrm{n}$ standard. The idea is to allow high data rate clients to aggregate certain number of frames (depending on a ratio between data rates of different clients) to mitigate air time unfairness. Though, such a balanced frame aggregation can compensate the throughput unfairness caused by different client's data rates it does worsen the response time which is critical for many real time applications like VoIP, etc [8].

Another possible approach is to induce the CSMA/CA media access control algorithm to giving priority to the clients depending on their data rates. This can be done, for instance, through the dynamic adaptation of the size of the contention window [9] that seems to be more promising as it does not affect the response time. Thus, in our further work we are going to investigate this approach in more details and find out the correspondence between the clients data rate and the desired size of the contention window.

\section{Acknowledgement}

We are grateful to Harald Richter for his feedback on the earlier version of this work. The work is partially supported by the FP7 KhAI-ERA project.

\section{References}

[1] JUN, P.: A Wireless MAC Protocol with Collision Detection, IEEE Transactions on Mobile Computing, vol. 6, No. 12, pp. 1357-1369, 2007.

[2] Impact of Legacy Devices on 802.11n Networks, Airmagnet Inc. Whitpaper, 2008, 15 p.

[3] PACHECO DE CARVAlHO, J. A. R., VEIGA, H., RIBEIRO PACHECO, C. F., REIS, A. D.: Performance Evaluation of Wi-Fi IEEE 802.11 A,G WPA2 PTP Links: a Case Study, Proc. World Congress on Engineering, 2012.

[4] KEMERLIS, V. P., STEFANIS, E. C., XYLOMENOS, G., POLYZOS, G. C.: Throughput Unfairness in TCP over WiFi, Proc. $3^{\text {rd }}$ Annual Conference on Wireless on Demand Network Systems and Services, 2003, pp. 1-6.

[5] MALONE, D., DUFFY, K., LEITH, D.: Modeling the 802.11 Distributed Coordination Function in Nonsaturated Heterogeneous Conditions, Networking, IEEE/ACM Trans. on, vol.15, No.1, 2007, pp.159-172.

[6] MALONE, D., DUFFY, K., LEITH, D.: Modeling the 802.11 Distributed Coordination Function in Nonsaturated Heterogeneous Conditions, IEEE/ACM Trans. on Networking, vol.15, No.1, 2007, pp.159-172.

[7] XYLOMENOS, G., POLYZOS, G. C., MAHONEN P., SAARANEN, M.: TCP Performance Issues over Wireless Links, IEEE Communications Magazine, vol. 39, No. 4, 2001, pp. 52-58.

[8] CAMPS-MURA, D., GOMONYB, M. D., PEREZ-COSTAA, X., SALLENT-RIBESC, S.: Leveraging 802.11n frame aggregation to enhance QoS and power consumption in Wi-Fi networks, Computer Networks, vol. 56, No. 12, 2012, pp. 2896-2911.

[9] YOUNGMI, J., KESIDIS, G.: Distributed Contention Window Control for Selfish Users in IEEE 802.11 Wireless LANs, IEEE J. on Selected Areas in Communications, vol. 25, No. 6, 2007, pp. 1113 - 1123. 